Obere Extremität 2020 $\cdot 15: 187-198$ https://doi.org/10.1007/s11678-020-00598-6 Published online: 19 August 2020 (C) The Author(s) 2020

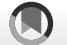

Check for
updates

Frank Gohlke' • Ayman A. Abdelkawi ${ }^{2}$. Hani Eltair ${ }^{1} \cdot$ Mohamed Aboalata' Walid Hussein ${ }^{1} \cdot$ Mohamed S. Abdrabo' ${ }^{1}$ Tina Jasper'

${ }^{1}$ Klinik für Orthopädie, Unfallchirurgie, Schulterchirurgie und Endoprothetik, Rhön-Klinikum Campus Bad Neustadt an der Saale, Bad Neustadt an der Saale, Germany

${ }^{2}$ Department of Orthopaedics and Traumatology, University of Assiut, Assiut, Egypt

\title{
Revision of failed reverse shoulder arthroplasty-a point of no return?
}

\section{Analysis of a series of 136 consecutive cases, review of the literature, and recommendations}

Due to the increasing number of reverse shoulder arthroplasty (RSA) implantations worldwide and the extension of the spectrum of indications [31], it is to be expected that the number of revisions after primary RSA is increasing even though the rate of complications is declining [2, $4,18]$.

In a long-term multicenter study of 1035 cases of Grammont-type RSA after a follow-up of more than 5 years, the authors observed infections in $3.6 \%$, humeral side in $3.2 \%$, glenoid side in $2.2 \%$, and neurological complications in $2.7 \%$ [6]. The rate of instability was reported as $2.3 \%$; additionally, there were stress fractures of the scapular spine in $1.8 \%$ of cases. The total rate of complications in this study was $11.5 \%$. This rate is much lower than the rate of complications in revision surgery of RSA, which is reported to be between 5 and $45 \%$ [3, 7, 15, 18, 28, 32].

Zumstein et al. [32] reported in their meta-analysis of RSA that $20 \%$ of the cases required revision. The authors defined the complications as any intraoperative or postoperative condition that has a negative impact on the outcome. They distinguished these cases from minor adverse findings unlikely to affect the result (e.g., scapular notching, postoperative hematomas, heterotopic ossifications, intraoperative cement leakage, and postoperative thrombophlebitis). Instability was the most common complication of RSA followed by infection, glenoid loosening, and glenosphere disassembly.

Corresponding to the variety of complications, five different categories of interventions can be distinguished:

- Revision surgeries in which the implant can be left in place unchanged in situ.

- Revision surgeries in which the prosthetic components are partially changed.

- Conversion of RSA to hemiarthroplasty or bipolar total shoulder prosthesis.

- Complete exchange of all components of the prosthesis in a one- or twostage procedure

- Resection arthroplasty in which all components are completely removed.

In general, it is not uncommon that the multifactorial cause of the complication is underestimated, misinterpreted, or even partially unrecognized [6]. This maylead to recurrence of complications after revision surgery. Therefore, it is strongly recommended to carefully analyze all risk factors, taking into consideration all aspects of clinical evaluation and imaging procedures as a basis for meticulous preoperative planning. This is imperative for successful revision surgery [3].
Ten years after the senior author performed his first revision cases of failed RSA, the authors believe that after the introduction of new designs and techniques, it is of interest to shed new light on the most common failure modes of RSA and to discuss the treatment algorithm for complications.

\section{Patients and methods}

The authors' experience with 392 revision arthroplasties performed using RSA between 2010 and 2019 was analyzed and 136 cases of revisions of RSA were identified, performed mainly by the senior author. Revisions of C-type periprosthetic fractures, in which conservative treatment or an open reduction internal fixation (ORIF) without touching the reverse implant was performed, were excluded.

The patients comprised 48 males and 88 females. Mean age of the patients at the day of revision was 68.3 years (29-88 years). In most of the cases (92\%) the primary RSA had been performed outside of the authors' hospital. Therefore, access to the preoperative X-rays and CT scans was limited. Furthermore, the first surgical report was available in only $11 \%$ of these cases. Moreover, the followup time was often short, and only 83 patients $(61 \%)$ could be examined before 
Table 1 Characteristics of the main pathology at revision, success rate, complications, and number and percentage of re-revisions

\begin{tabular}{|c|c|c|c|c|c|}
\hline Primary indication & $\begin{array}{l}\text { Number, } \\
(\%)\end{array}$ & $\begin{array}{l}\text { Success rate (free of pri- } \\
\text { mary pathology or signifi- } \\
\text { cantly improved; } n \text { ) }\end{array}$ & $\begin{array}{l}\text { Staged } \\
\text { revisions } \\
(n)\end{array}$ & $\begin{array}{l}\text { Complications } \\
\text { (intra-/ } \\
\text { postoperatively) }\end{array}$ & Unplanned re-revision \\
\hline Instability & $24(17.6 \%)$ & 19 & 1 & $\begin{array}{l}4 \text { re-dislocations, } \\
1 \text { persistent } L G \text { infec- } \\
\text { tion }\end{array}$ & $\begin{array}{l}1 \times n=4,2 \times n=2,1 \times \text { in } \\
n=3, \text { total } 11 \text { in } 7 \text { pa- } \\
\text { tients }(29.2 \%)\end{array}$ \\
\hline Nerve lesion & $7(5.1 \%)$ & 5 & 2 & & - \\
\hline Humeral loosening & $43(31.6 \%)$ & 42 & 13 & $\begin{array}{l}\text { Dislocation, peripros- } \\
\text { thetic fracture }\end{array}$ & $n=3(7 \%)$ \\
\hline Glenoid loosening & $26(19.1 \%)$ & 21 & 12 & $\begin{array}{l}\text { Aseptic loosening, } \\
\text { glenoid neck frac- } \\
\text { ture, dislocation }\end{array}$ & $n=5(20 \%)$ \\
\hline $\begin{array}{l}\text { Technical failure, e.g., baseplate } \\
\text { malposition, excessive anteversion/ } \\
\text { retroversion, + design failure }\end{array}$ & $10(7.4 \%)$ & 9 & - & - & - \\
\hline Stiffness/heterotopic ossification & $6(4.4 \%)$ & 4 & - & $\begin{array}{l}2 \times \text { recurrence of } \\
\text { arthrofibrosis }\end{array}$ & - \\
\hline Periprosthetic infection & $29(21.3 \%)$ & 28 & 25 & $\begin{array}{l}1 \times \text { persisting re-in- } \\
\text { fection }\end{array}$ & $n=4(16.7 \%)$ \\
\hline $\begin{array}{l}\text { Periprosthetic fracture (cases stabi- } \\
\text { lized by ORIF or strut grafts, without } \\
\text { revision of the components ex- } \\
\text { cluded) }\end{array}$ & $21(15.4 \%)$ & & 2 & $\begin{array}{l}\text { Removal of cerclage } \\
\text { wires, additional } \\
\text { bone grafting }\end{array}$ & $n=2(9.5 \%)$ \\
\hline \multirow[t]{2}{*}{ Scapula spine fracture } & $6(4.4 \%)$ & 3 & - & $\begin{array}{l}\text { Plate exchange, } \\
\text { partial removal }\end{array}$ & $n=2(33.3 \%)$ \\
\hline & Total: 136 & $\begin{array}{l}\text { Total: } 120 \\
88.2 \%\end{array}$ & $\begin{array}{l}\text { Total: } 55 \\
40.4 \%\end{array}$ & & Total: 27 (19.9\%) \\
\hline \multicolumn{6}{|c|}{ ORIF open reduction internal fixation, $L G$ low-grade } \\
\hline
\end{tabular}

the last control at a minimum of 2 years, whereas $35 \%$ could be examined within 9 years.

We grouped our patients according to their main indication for the first revision as "primary diagnosis" and analyzed the complete pre- and intraoperative pathology, noted as "concomitant" diagnosis. The surgical technique, type of implant, and rate of further revisions and complications were evaluated. The patients were postoperatively followed up clinically and radiographically at 3 months, 6 months, 1 year, and annually thereafter.

\section{Results}

\section{Primary indications}

The main indication for revision surgery was chronic infection in 34 patients $(25 \%)$ and instability in 24 patients (17.6\%). Another 21 patients (15.4\%) were admitted and operated on for periprosthetic fractures. Of these, 10 patients had stable implants while there was radiological evidence of stem loosening in 11 patients, of whom 8 had positive biopsies and cultures postoperatively proving low-grade infections.

Aseptic loosening of the stem without preoperative evidence of infection was the main indication in 15 patients (11\%). In 3 patients (2.2\%) revised for instability as the main indication and without abnormal radiographic findings on the humeral side, the stem was found to be loose and/or in excessive anteor retroversion intraoperatively. Concomitant stem loosening was observed in 25 patients $(18.3 \%)$ revised primarily for chronic infection.

Aseptic loosening of the baseplate was the main indication for revision in $23 \mathrm{pa}$ tients $(16.9 \%)$. In addition, 3 cases were intraoperatively identified during the revision of instability and excessive notching.

Progressive and painful scapular notching due to malposition of the glenosphere was the reason for revision in 4 patients $(2.9 \%)$ and excessive polyethylene (PE) wear of the PE liner without significant malposition in 2 further cases $(1.5 \%)$.

Fractures of the scapular spine were operated on in 6 cases (4.5\%), 2 after a significant trauma and 4 fatigue fractures which failed after conservative treatment.

Rare indications for revision were nerve lesion $(n=3,2.2 \%)$ caused by prominent screws with evidence for nerve compression, and in 4 cases the axillary nerve or brachial plexus was revised after iatrogenic lesions which did not recover completely combined with exchange of components for instability and periarticular release of severe stiffness.

Removal of heterotopic ossifications, displaced tuberosities, and disrupted cables was associated with severe stiffness and was revised in 6 patients, all cases posttraumatic (4.4\%).

According to the type of intervention (- Table 1), revision without changing the components was performed in only 8 cases, while 54 cases required partial exchange of modular components. In the majority of patients, complete re- 
Obere Extremität 2020 · 15:187-198 https://doi.org/10.1007/s11678-020-00598-6

(c) The Author(s) 2020

F. Gohlke · A. A. Abdelkawi · H. Eltair · M. Aboalata · W. Hussein · M. S. Abdrabo · T. Jasper

\section{Revision of failed reverse shoulder arthroplasty-a point of no return? Analysis of a series of 136 consecutive cases, review of the literature, and recommendations}

\section{Abstract}

Background. The rate of complications after revision of reverse shoulder arthroplasty (RSA) is higher than it is in primary RSA, depending on the type of surgical intervention, the follow-up time, the preoperative condition of the patient, and the experience of the surgeon.

Objective. The current article represents an evaluation of the authors' experiences with revisions of RSA and a review of literature, in order to define prognostic parameters and surgical options for the most common modes of failure.

Materials and methods. Between 2010 and 2019, 136 revisions of RSA were performed. Mean age of the patients at surgery was 68.3 years (29-88 years). The main indication was instability in 24 patients and chronic infection in 34. Aseptic loosening of the stem was the main indication in 15 and aseptic loosening of the baseplate in 23 patients. Periprosthetic fractures were present in 21 patients. Fractures of the scapular spine were operated on in 6 and surgery was performed for progressive notching due to malposition of the glenosphere in 4 patients. Results. Staged procedures, use of allografts, and custom-made implants are common, especially for chronic infections and severe bone loss. Most of the patients (88.2\%) were treated successfully. Two salvage procedures and two retentions of spacers occurred. We identified risk groups for re-revision: chronic dislocations, chronic infections, advanced bone loss, and scapular spine fractures. We observed a shift of indications and techniques, often related to the design of the implants. Conclusion. These results confirm that careful preoperative planning, special implants, and a high level of experience are mandatory. With time, the authors developed an algorithm for certain indications because it was recognized that failures are often of multifactorial origin. Today, numerous implant designs which differ considerably in biomechanical features and failure modes are available. This makes revisions of RSA more complex than it was in the past.

\section{Keywords}

Reoperation, Revision · Risk factors · Arthroplasty, replacement, shoulder - Shoulder joint . Reverse shoulder arthroplasty

\section{Revisionen fehlgeschlagener inverser Schultertotalendoprothesen - kein Weg zurück? Analyse einer Serie von 136 eigenen Fällen, Übersicht der Literatur und Empfehlungen}

\section{Zusammenfassung}

Hintergrund. Die Komplikationsrate bei Revisionen inverser Schultertotalendoprothesen („reverse shoulder arthroplasty", RSA) ist gegenüber primären Implantationen deutlich erhöht - abhängig von Indikation, Operationsverfahren, Beobachtungszeitraum sowie den Ausgangsbedingungen und der Erfahrung des Chirurgen.

Ziel der Arbeit. Die Zielsetzung dieser Arbeit besteht in einer Analyse eigener Erfahrungen mit Revisionen von RSA, um unter Berücksichtigung der aktuellen Daten in der Literatur prognostische Parameter und Behandlungsstrategien für die häufigsten Komplikationen zu erarbeiten.

Material und Methoden. Zwischen 2010 und 2019 wurden 136 Revisionsoperationen an RSA durchgeführt. Das mittlere Alter der Patienten betrug 68,3 Jahre (29 bis 88 Jahre). Primäre Indikationen für den
Eingriff waren Instabilität bei 24 und eine chronische Infektion bei 34 Patienten. Aseptische Lockerungen stellten bei 23 , periprothetische Infektionen bei 21, Frakturen der Spina scapulae bei 6 und progressives "Notching" aufgrund einer Fehlimplantation der Basisplatte bei 4 Patienten die primären Indikationen dar.

Ergebnisse. Zweizeitige Eingriffe, die Verwendung von Allografts und Sonderanfertigungen waren häufig, insbesondere nach chronischen Infektionen und bei ausgeprägten Knochendefekten. Die meisten Patienten (88,2\%) wurden erfolgreich behandelt. Rückzugsoperationen (2 Konversion auf Hemiprothese oder 2 Resektionsarthroplastiken) waren selten. Es konnten folgende Risikogruppen mit erhöhter Rerevisionsrate identifiziert werden: chronische Instabilität, chronische Infektionen, erhebliche Knochendefekte sowie Frakturen der Spina scapulae. Innerhalb der letzten 10 Jahre waren Verlagerungen der Indikationen und Operationstechniken zu verzeichnen, die oft im Zusammenhang mit dem Implantatdesign standen.

Schlussfolgerung. Eine sorgfältige präoperative Analyse und Operationsplanung sowie langjährige Erfahrung sind Voraussetzungen für die erfolgreiche Behandlung. Da häufig mehrere Ursachen bei einem Implantatversagen vorliegen, wurde ein Algorithmus für die wichtigsten Indikationen entwickelt, der kontinuierlich an neue Implantattypen und Operationstechniken angepasst werden muss.

\section{Schlüsselwörter}

Reoperation, Revision · Risikofaktoren · Arthroplastik, Ersatz, Schulter · Schultergelenk . Inverse Schulterendoprothese moval of all components and replacement with new implants was necessary $(n=73$, $54 \%)$, often performed as staged surgery in two $(n=44,32 \%)$ or more $(n=11,8 \%)$ preoperatively planned steps.

Salvage procedures such as resection arthroplasty or conversion to hemiarthroplasty were rarely needed $(n=2$,
$1.5 \%)$ and mostly due to severe comorbidity of the patient. Nine patients rejected further recommended surgery because of significant comorbidities or sufficient pain relief despite limited function, especially after implantation of articulating spacers $(n=2)$.
Within the follow-up of mean 4.2 years (1-9 years), besides the 55 planned staged procedures, 27 (19.8\%) revisions were observed, 12 caused by recurrent dislocation, 5 caused by recurrent aseptic glenoid loosening, 4 related to periprosthetic infections (only one re-infection), 2 after plating of a scapular spine re- 
fracture caused by non-union, 2 related to periprosthetic fractures after a delay of more than 6 months, and 2 revisions for minor events (hematoma, breakage of wires).

\section{Instability}

Risk factors for instability in the current patients were insufficiency of the soft tissues such as damage of the deltoid muscle and pectoralis major, complete insufficiency of the rotator cuff (especially the absence of subscapularis and teres minor), and injuries of the axillary nerve or brachial plexus. Moreover, neurological disorders such as Parkinson's disease, incompliance due to dementia, cerebral ischemia, seizures, or chronic alcoholism interfered with soft tissue or implant-related problems.

The most common skeletal causes of instability were severe humeral bone loss; medialization of the glenoid due to excessive wear and abnormal version and inclination; and displaced fractures of the coracoid and acromion or the scapular spine, which lead to a decrease of the compression joint load.

Technical problems included inadequate reconstruction of the humeral length, excessive anteversion or retroversion $>20^{\circ}$, use of too-small components, superior positioning or tilting of the baseplate $[9,27]$, glenoid medialization, and mechanical impingement. Superior inclination exceeding more than $15^{\circ}$ and malposition of the baseplate may result not only in subtle instability [27], but also in rapidly progressive notching and increased shear forces [9], especially in adduction and at the endpoint of rotation. Both may also provoke early pullout of the baseplate and displacement. Therefore, we recommend a careful analysis of X-rays taken in standard planes and of the entire humerus, or even bilateral CT imaging for precise bilateral measurements of humeral length as well as for assessment of the position and size of the components. Additionally, infection, especially after multiple previously performed surgeries, should be excluded by taking blood samples for C-reactive protein (CRP) and sedimentation rate.
In the 24 patients operated on for instability, mostly for long-standing dislocations of more than 3 months, a total of 12 revisions were observed in 8 patients, once in four cases of recurrence, two patients twice, and one patient three times. Additionally, one dislocation occurred after revision of infection and stem loosening. All failures occurred with a delay of more than 6 weeks after revision and were associated with neurological disease (e.g., partial axillary nerve palsy, Parkinson's disease); low-grade infection $(n=3)$; or use of crutches, wheelchair, or walking frame in severe walking disability; therefore, mostly of multifactorial origin. Open reduction was required in all cases and needed the exchange of one $(n=9)$ or more components on both the humeral and glenoid side $(n=15)$. However, two or more re-revisions were necessary in 7 patients, performed as additional humeral lengthening by modular components or stem exchange.

\section{Chronic periprosthetic infection}

In general, the rate of infection after primary RSA seems to be higher than in primary anatomical shoulder endoprosthesis, 2.9 versus $0.51 \%$ are described by Bohsali et al. [2]. Moreover, the rate of infection in revision RSA is even higher and reported to be $5.8 \%[3,4,32]$.

The diagnosis of infection was obvious in all cases with a chronic sinus, enhanced CRP values, and multiple prior revisions outside the authors' institution for any problem like pain, stiffness, or instability confirmed by positive cultures of a pathogen after biopsy.

In all cases of revisions, at least six biopsies are routinely taken intraoperatively from different regions and for different cultures in order to exclude hidden low-grade infections. Loosening of any components, stem or baseplate, is only regarded as "aseptic" once the final result of the cultures after 2 weeks is negative and there is no pathological finding in histology.

The most common pathogens in the present 34 cases of chronic infections were, corresponding to the literature, low-grade pathogens such as Cutibacterium acnes (formerly Propi- onibacterium acnes) and Staphylococcus epidermidis. In the remaining 103 "aseptic" cases of revision, unexpected positive cultures and histology of low-grade pathogens were found intraoperatively in 15 patients $(17.5 \%)$, with almost the same spectrum as in the revisions of chronic infections: predominantly $C$. acnes (40\%), S. epidermidis (20\%), and S. warneri (13\%). Preoperatively these 15 patients did not show clinical evidence of infection, with normal or borderline CRP values.

The infection rate increased with the number of previously performed surgeries in fractures, multiple repair of shoulder instability or failed rotator cuff repair, and revision of anatomical arthroplasty to RSA [4]. An association with the hollow space under the glenosphere or a superior approach via delta split, particularly for C. acnes, is discussed.

Failed revision arthroplasty is regarded as a predisposing factor for lowgrade infection [3-5]. Only 77 (56.6\%) patients experienced only one surgery before the current revision (mean 1.7 revisions each). The remaining 59 patients had a history of at least two previous revisions (22 of them had more than 4 ).

Only one of our cases was an acute hematogenous infection 5 years after primary RSA. This was successfully treated with debridement and exchange of the glenosphere and PE liner along with systematic antibiotic therapy. In most chronic infections the implant was completely removed in a two-stage procedure, first using an articulating antibiotic-loaded spacer besides systemic antibiotic therapy, followed by reimplantation of the RSA after eradication of infection at least 8 weeks later. However, removal of implants during revision surgeries carries a high risk of periprosthetic humeral fracture and secondary bone loss.

Exchange of the components can be successful if the pathogen is already known before surgery and not resistant to antibiotics. However, in the literature, most surgeons prefer two-staged revisions with antibiotic-loaded articulating spacers. Besides the chance of a more radical debridement, this step provides 

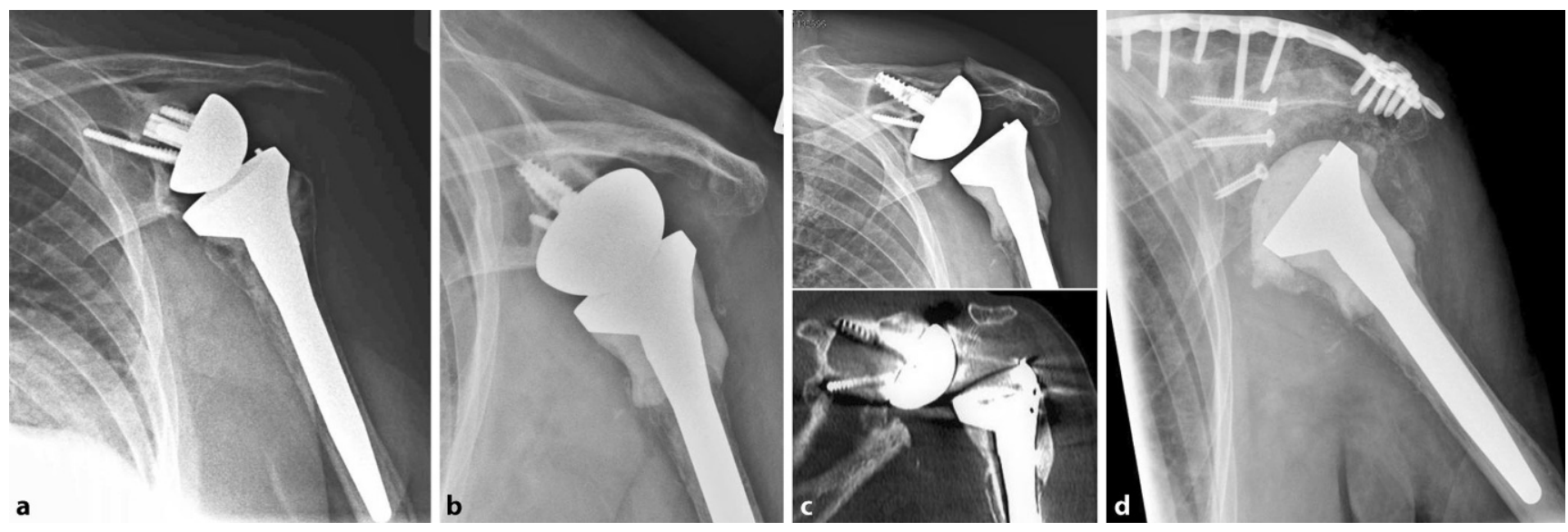

Fig. $1 \Delta$ Aseptic glenoid loosening in a female rheumatoid arthritis patient and recurrence after 2 years. a 2004; implantation of reverse shoulder arthroplasty (RSA) in a 65-year-old patient, posterior-superior cavitary bone loss. She developed progressive loosening of the baseplate, which was revised in a staged procedure with reconstruction and partial removal of the humeral component. b After 12 weeks, the patient was revised with a baseplate with a central screw. CX-ray and CT scan taken after 2 years. Unfortunately, the screw was not coated, therefore bone ingrowth had not occurred. She presented herself with moderate pain and complete loss of active elevation but no signs of infection. The anteroposterior X-ray shows excessive bone loss $5^{\circ}$ and fatigue fracture of the scapular spine and coracoid base. $d$ The patient was scheduled for staged re-implantation. In the first step, the glenoid was reconstructed with an allograft. The scapular spine was plated and morselized allograft added. The patient rejected further surgery because of poor health

the opportunity for detailed evaluation of bone loss and better planning of suitable revision implants, augments, and bone grafts needed for reconstruction in the final step. The authors prefer the use of staged revision surgery, particularly in the presence of biofilm-producing or multiresistant pathogens. The success rate in terms of eradication of the infection was $97 \%$ in the present series.

The authors do not recommend resection arthroplasty, even as a salvage procedure, because the functional result usually remains unsatisfactory [7]. Some of the current cases with low-grade infection were associated with recurrent instability and major bone loss on the glenoid and humeral side. In this series, this association prove to be a significant poor outcome factor for both enhanced risk of re-intervention and a limited functional result.

\section{Nerve injuries}

Recent studies demonstrate an overall rate of nerve lesions of $1.2-14 \%[3,4,13$, 32] in patients after RSA, but revisions are rare due to recovery in most of the cases [17]. The rate of nerve lesions seems to be higher in RSA than in anatomic shoulder prosthesis $[3,4,13,15,17,20]$, related to the tendency of arm lengthening and over-tensioning of the soft tissues [17, 20]. Isolated axillary nerve lesions are the most frequently encountered lesions, followed by brachial plexus injuries [25]. The majority of nerve injuries have a good chance of recovery [17]. Risk factors for development of permanent nerve injuries include excessive overlengthening of the arm of more than $40 \mathrm{~mm}$. This may occur more frequently with bulky platform systems, especially in an "onlay design" rather the classic "inlay design" of the Grammont style, in which the concavity of the metaphyseal part of the humerus is below the humeral head resection line. In two cases a partial exchange of components (shortening of the humeral length combined with lateralized glenosphere) was performed and in three removal of penetrating screws in the suprascapular nerve or brachial plexus in addition to decompression of the axillary nerve and brachial plexus.

\section{Fractures of the scapular spine}

The prevalence of this complication is estimated to be $1-5 \%$, on average $2.8 \%$ [ 1 , $3-5,13,21]$, depending on the patient and implant type. Both the lateralized glenosphere design or platform systems with an onlay design [1] are reported to cause significantly higher rates in comparison to the classic Grammont design, probably due to excessive lateralization of both glenoid and humeral sides and, rarely, the placement of the superior screw of the baseplate. Initially, minor displaced spine fractures are often missed in plain $\mathrm{X}$-rays and need a CT scan for diagnosis.

In the authors' experience, healing of this type of fracture can be achieved by conservative treatment in up to $50 \%$ of cases if it is immediately treated by immobilization in an abduction brace. Administration of vitamin $\mathrm{D}$ and immediate halting of bisphosphonate medication is mandatory.

Attempts to stabilize these fractures with plates are often associated with loosening and fatigue of plates, poor functional outcome, and a high rate of pseudarthrosis of at least $50 \%$ [21]. Isolated scapula spine fractures were seen in 2 patients of the current series and an association of glenoid loosening and advanced bone loss $4-5^{\circ}$ with fatigue fracture of the base of scapular spine and the coracoid process in 3 patients ( $\bullet$ Fig. 1 ). The revision required autograft and allograft reconstruction of the glenoid in a twostage procedure together with plating of the scapular spine. 


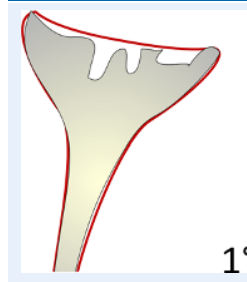

Mild bone loss (centered or eccentric $<15^{\circ}$, loss of $<20 \%$ of native contact area of the glenoid surface)

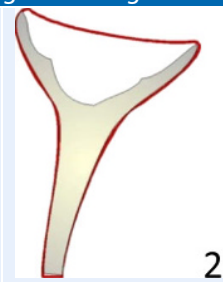

Moderate bone loss, the Severe eccentric (periphglenoid vault is intact

f

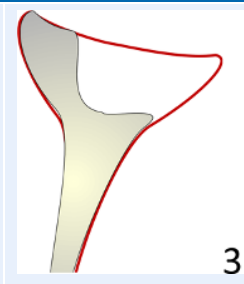
eral) bone loss ( $>20^{\circ}$ verglenoid vault is not intact; one wall is broken or defi-
All types of baseplate
feasible

All types of baseplate
feasible

Long-post baseplate, transfixation technique + bone graft (preferably substantial block) sion or $50 \%$ of width), the cient

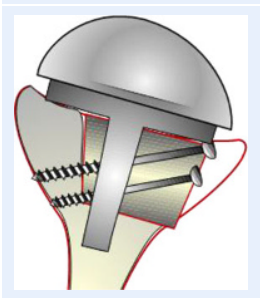

Long-post baseplate + auto/allograft, 1- or 2-stage procedure, depending on primary fixation strength

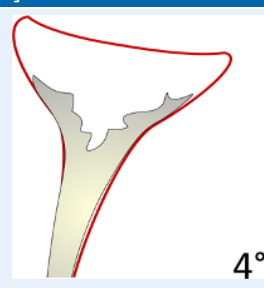

Severe bone deficiency and advanced medialization of both posterior and anterior wall of the glenoid vault. Remaining native bone stock provides $>10 \mathrm{~mm}$ fixation of the post

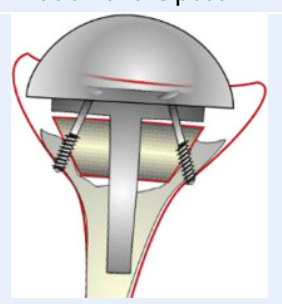

Transfixation technique + structural bone graft in 1- or 2-stage procedure, reconstruction of joint line mandatory

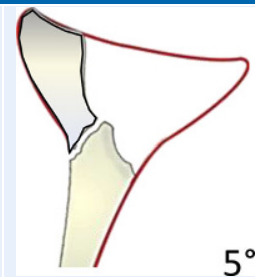

Severe bone deficiency, excessive medialization, and/or breakage of both posterior and anterior wall of the glenoid vault. Remaining native bone stock $<10 \mathrm{~mm}$

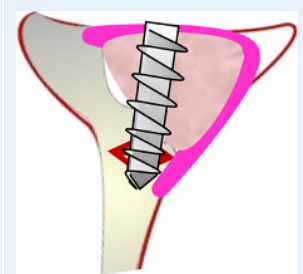

Two-stage procedure with bulk auto-/allograft or custommade implant with vault filling wedge

\section{Aseptic baseplate loosening}

Aseptic loosening of the baseplate of the RSA is a rare complication compared to loosening of the glenoid component in anatomic shoulder arthroplasty. Even in long-term follow-up, it accounts for only $1.7 \%$ of cases [6]. Therefore, a lowgrade infection as the cause of early loosening should be excluded. Early loosening of the baseplate (during the first weeks postoperatively) suggests inadequate strength of the primary anchorage because of a technical failure or poor quality of bone stock. Malposition of the baseplate (superior inclination exceeding $20^{\circ}$ or more than $20 \mathrm{~mm}$ above the inferior glenoid rim) as well as too much lateralization can lead to excessive shearing forces and pull-out of the baseplate $[3,9,27]$.

If a bone graft has been used, loosening may occur due to inadequate fixation of the central post or screw in the native bone (which should exceed at least $5-10 \mathrm{~mm})[11,12]$. We introduced the "three-column concept" [12] for adequate fixation even in severe glenoid bone loss. The individual construct of a baseplate with a central coated post, angu- lar locking screws, and substantial bone graft is allocated to each pattern of bone loss in our classification (•Table 2). This classification provides an algorithm for selection of the most appropriate surgical procedure. In principle, the glenoid reconstruction can be performed in a oneor a two-stage procedure with autograft (iliac crest) or allograft (femoral head) in a modified transfixation technique. The authors recommend making the final decision intraoperatively based on the fixation strength that could be achieved for baseplate fixation. Baseplates with a central (non-coated) screw instead of a coated post provide the surgeon with the feeling of a strong "grip" during surgery, but may fail with a delay of more than 6-12 weeks ( $\bullet$ Fig. 1) because of lack of bone ingrowth, particularly in bulk allografts [24]. In the authors' own series of 145 cases, operated on revision cases suffering from type $2-5^{\circ}$ glenoid bone loss, an overall success rate of more than $90 \%$ was found for both one- and twostage reconstructions. This was mainly related to the high success rate of autograft incorporation, taken from the iliac crest, comparable to the majority of data published in the literature [11, 12].
Late baseplate loosening in RSA was mostly associated with severe uncontained bone loss (type $4-5^{\circ}$ ) in the present series, sometimes combined with fatigue fractures of the scapular spine and coracoid process due to destruction of the glenoid vault by the central screw or post ( $\bullet$ Fig. 1). Twostage procedures allow a "second-look" CT scan after removal of the implant, which is important for planning the final reconstruction. Small cannulated titanium screws used for bone graft fixation provide the surgeon with valuable information regarding the best position of the post and the correction of glenoid version and inclination.

Excessive bone loss in patients who are in bad health or reject further surgery may require salvage procedures such as conversion to hemiarthroplasty or complete removal of hardware, or resection arthroplasty. In the literature, the clinical results for both are disappointing [7]. Among the present patients there was fortunately only one case of conversion to hemiarthroplasty. This patient rejected glenoid reconstruction or any major surgery because she was a Jehovah's Witness and therefore scared of any 

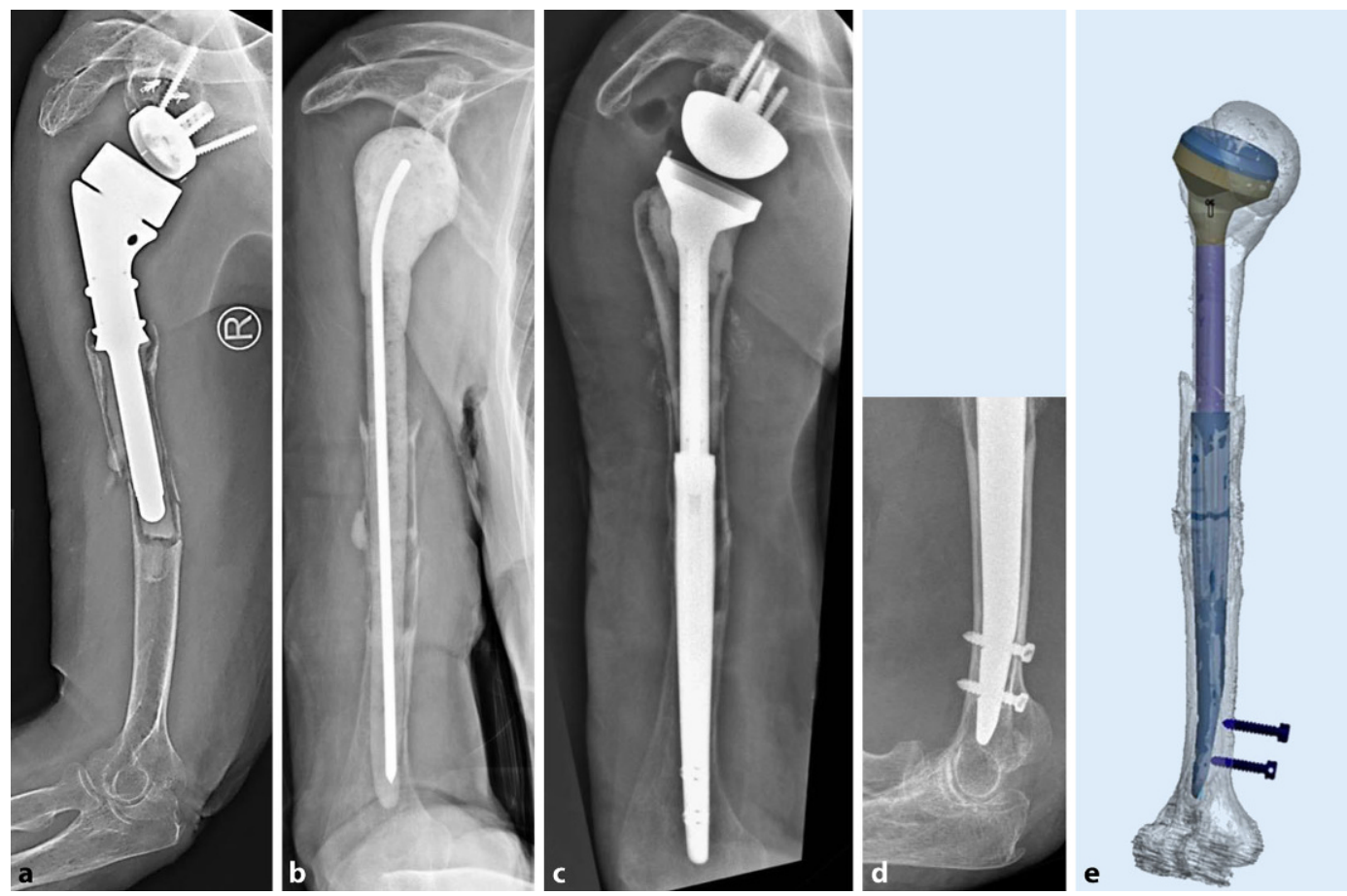

Fig. $2 \Delta$ Staged revision of failed reverse tumor arthroplasty with multifactorial pathology: instability, low-grade infection, stem loosening, and periprosthetic fatigue fracture. a 84-year-old female patient with multiple previous surgeries after failed treatment of proximal humerus fracture and hemiarthroplasty. She was revised at a different location to reverse tumor arthroplasty, twice revised for recurrent dislocations outside the authors' hospital. $b$ In a first step, the tumor prosthesis was removed and replaced by an antibiotic-loaded, articulating spacer. $\mathrm{C}$ Due to humeral bone loss $4^{\circ}$ and glenoid bone loss $4^{\circ}$ after removal of the infected base plate, she was revised to a custom-made modular stem with distal bent, distal interlocking screws, and additional humeral allograft, both measured with bilateral CT scan. $\mathbf{d}$ Lateral X-ray of the elbow after 2 years. e Preoperative 3D reconstruction of the custom-made implant

blood loss. Two patients rejected further surgery after the first step with allograft reconstruction combined with a humeral spacer.

Technical and design-related failures were observed in 10 patients (7\%). Comparing to the authors' own experience before 2010, disassembly of the glenosphere was a rare condition. This is due to improvement of old designs, applying both the technique of cold welding and additional assembly screws in order to prevent dissociation.

\section{Scapular notching}

The clinical relevance of scapular notching with a stable base plate (grades 1-3) is controversial. The rates were between 44 and $96 \%$ in the first series of patients [18, 21]. Modern implants and improved surgical techniques (proper inferior placement without superior inclination) have recently reduced the rate of severe notching (grades 3-4) to $0-10 \%[3,4,9]$.
Inferior notching initially appeared to have little effect on the clinical outcome in mid-term follow-up [19], but in the authors' experience it affects the function and the rate of late baseplate loosening by progressive osteolysis after more than 5-10 years. Intraoperatively, advanced osteolysis was mostly found behind the posterior-inferior rim of the glenoid.

Strategies to improve notching include exchange to a larger or eccentric glenosphere, lateralization of the center of rotation, or use of reversed shoulder arthroplasty with a neck shaft angle reduced to $130-135^{\circ}$. The lack of availability of implants designed more than 10 years ago limits this last option for revision. In the authors' experience, excessive notching was often caused by a malposition of the baseplate in superior inclination of more than $10^{\circ}$, fixation in a superior position, and the selection of a too small glenosphere diameter in male patients, which resulted in a poor active range of motion, pain, and rapidly progressive notching [9]. The exchange to a larger and/or eccentric glenosphere and higher PE liner adapted to the new glenosphere size provided substantial improvement of pain and function, even when the wellfixed baseplate was left in place.

\section{Humeral side revisions}

\section{Periprosthetic fractures}

Periprosthetic fractures can be induced by stress shielding, osteolysis, and fatigue in stem loosening, or initiated by cracks and bone loss which occur during stem extraction in revisions. The management of these fractures depends on the etiology, the remaining bone stock, and the fracture type. In the current patient series, stem loosening (11 cases, $54 \%$ ) and chronic low-grade infections (34\%) were concomitantly found in a high proportion of patients admitted to the hospital for acute periprosthetic fractures.

Management of these fractures has to consider the location of the fracture 

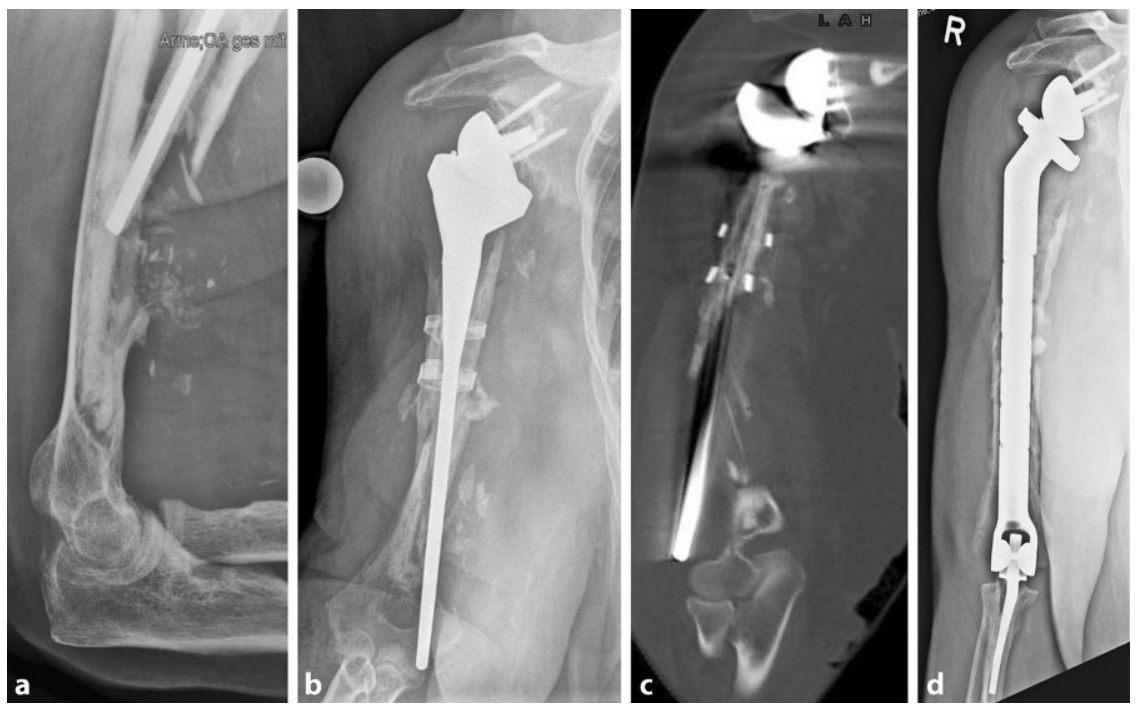

Fig. $3 \Delta$ Bipolar reverse tumor arthroplasty in a 74-year-old female patient with diabetes and chronic lymphocytic leukemia. a X-ray taken 1 year after revision of failed reverse shoulder arthroplasty (RSA) performed at a different location for stem loosening and low-grade infection with stem perforation, loosening, and filling of the medullary canal distally close to the elbow.b X-ray of the elbow shows bone loss type $5^{\circ}$.cCT scan shows destruction of the humeral bone stock preoperatively. $\mathrm{d}$ Revision to bipolar tumor arthroplasty and cerclage fixation of bone fragments by suture cerclage after 2 years

(metaphyseal, mid-shaft or distal diaphyseal, supracondylar), the fracture type and pattern (one or multiple fragments, transverse, long spiral, etc.), the bone quality, and the stability and type of the previously implanted prosthesis. In case of stem loosening, the authors prefer the exchange to a longer stem in addition to fixation with cables or cerclages. In type $\mathrm{C}$ and stable stem, the treatment of choice was a long pre-contoured cable plate from a posterior approach. Difficult cases with poor remaining bone stock and insufficient anchorage length in the distal humerus (type Cor with bone loss $4^{\circ}-5^{\circ}$ ) required long modular revision stems with supracondylar interlocking screws and distal bent for cementless fixation (• Fig. 2).

Basically, the intramedullary fixation with a long-stemmed prosthesis is generally more stable and often easier to perform than other types of fixation using cable plate fixation with/without additional strut allografts. External fixation has, in the authors' opinion, a much higher failure rate, as reported in the literature, which is probably related to poor vascularity of the remaining bone stock in the long run.

Minimally displaced long spiral fractures with well-fixed stable stems can be treated conservatively, but this requires a long period of immobilization of about 3-4 months which is rarely tolerated by the patients.

In case of complete destruction of the humeral bone stock, tumor arthroplasty with bipolar joint replacement of both the shoulder and the elbow was used (- Fig. 3). Unfortunately, the proper length of the humerus and soft tissue tensioning, which is required to keep the reverse implant stable but allows at least $100^{\circ}$ of elbow flexion, is intraoperatively difficult to adjust. In order to preserve attachment of the soft tissues, it is mandatory to fix diaphyseal eggshell fragments to the body of the tumor prosthesis or to cover it completely with Dacron ${ }^{\circledR}$ (DuPont, Wilmington, NC, USA) mesh. Moreover, the patients who needed tumor replacement were elderly and had severe comorbidities such as cardiovascular insufficiency, diabetes, and dementia. Two patients deceased 5 and 10 weeks after the revision surgery, one due to cachexia and pneumonia, the second after a complication of her alcoholic liver disease.

The authors thus strive to avoid tumor replacement because the revision of five failed reverse tumor prostheses were experienced to be very demanding proce- dures, due to the high rate of re-revision and associated problems (recurrent dislocations, advanced bone loss, soft tissue deficiency, secondary revisions for loosening, periprosthetic fatigue fractures). In all failed tumor arthroplasties, some ingrowth of scar tissue in the Dacron mesh was found intraoperatively, but was unfortunately displaced in delamination from the metal body after the rupture of cerclage sutures.

\section{Stem loosening}

Melis et al. [22] observed progressive osteolysis in the proximal humerus, especially around the tuberosities, and stem loosening in $8.8 \%$ of the patients on average 10 years after primary implantation of RSA.

Cases of stem loosening admitted to the authors' hospital were mainly failed fracture or revision RSA, with obviously poor cementation techniques or cementless fixation. Because early "aseptic" stem loosening is always suspect for low-grade infections, samples (both histology and cultures) should be taken when in doubt (borderline enhanced CRP values) by arthroscopy prior to open revision.

However, in a recently performed long-term multicenter study in France [6], aseptic loosening of the stem was not more common than baseplate loosening. However, after 5.3 years on average, complete humeral radiolucency was observed in $16 \%$ of the cases, leading to subsidence of the stem in $1.3 \%$ and partial or complete osteolysis of the tuberosities in $60 \%$. The authors interpret this as the result of both stress shielding and severe $\mathrm{PE}$ wear or notching. In the current series of cemented long-stemmed revision implants used for revision of anatomical hemiarthroplasty, humeral subsidence was found in $11 \%$ of the patients after an average of 7 years of follow-up [30]. Therefore, it was concluded that a new design with cementless modular stems is needed for revisions, even when it must be conceded that in the current study, cemented revision stems were used in an unfavorable selection of elderly, mostly female patients with severe osteoporosis and proximal bone loss.

The conversion of "stemless" RSA with metaphyseal fixation or short stems 
Table 3 Treatment algorithm according to the classification of Gohlke and Werner [10]. The amount of detachment of muscle insertions dictates the use of humeral allografts according to the type of humeral bone loss. In type 5 , the remaining bone stock does not provide the minimal anchorage length for fixation of a standard revision stem

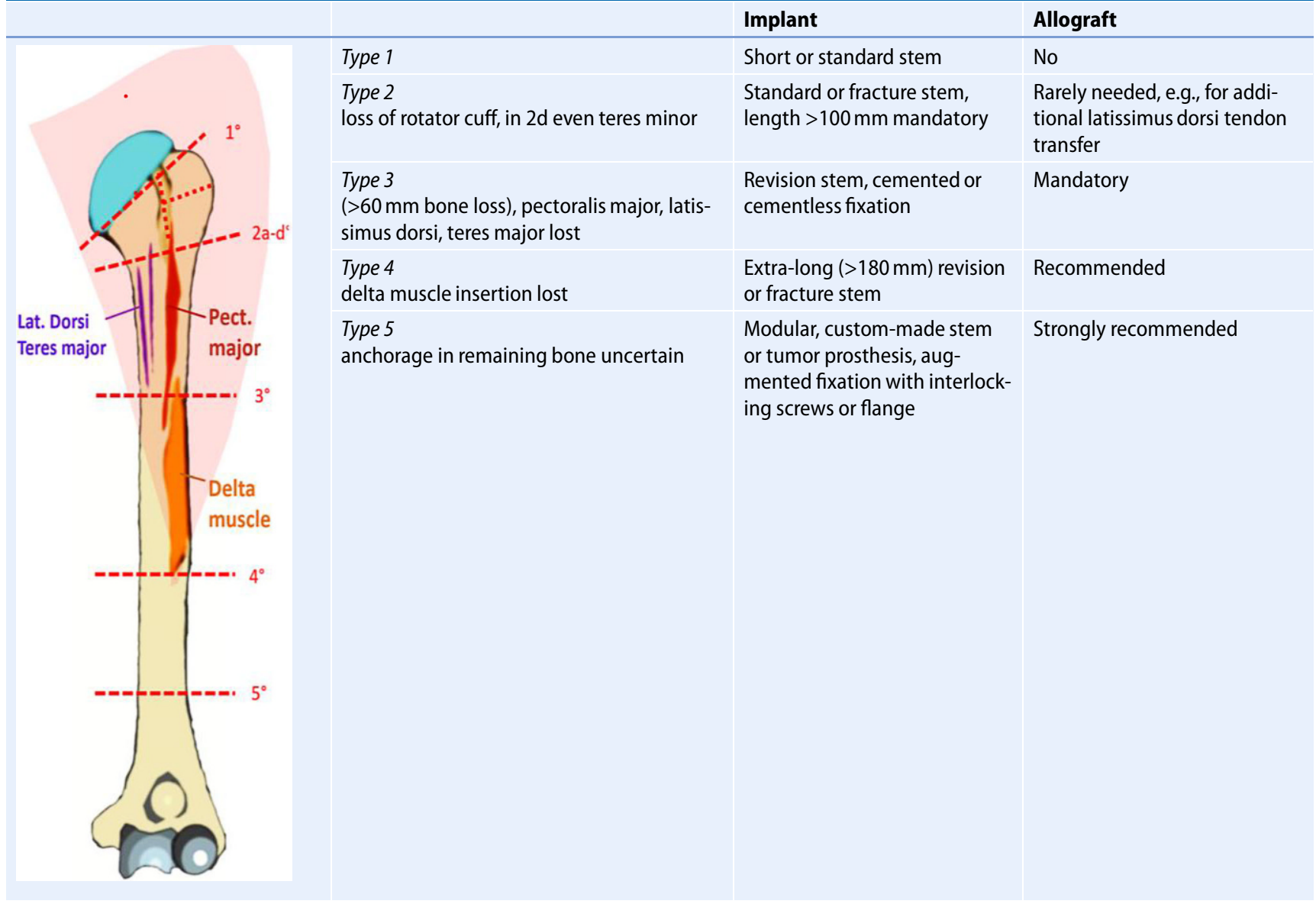

is comparatively easy to perform, while conversion of a well-fixed long-stemmed RSA is a more demanding procedure and carries the risk of intraoperative periprosthetic fracture and increased humeral bone loss. The conversion of modular platform systems [29] is obviously less time consuming and blood sparing, but, unfortunately, this is only available as an option in $20-30 \%$ of the cases.

The authors have been using their previously published classification of humeral bone loss as a guideline to choose the correct length of the revision implant and to evaluate the need for a humeral allograft since 2009 [11, 12]. This is more orientated to the detachment of muscle insertions than the length of humeral bone loss (- Table 3). Allografts are considered in the case of more than $50 \mathrm{~mm}$ bone loss. If the humeral bone loss extends inferior to the insertion of the deltoid muscle (more than 50\% humeral length), a modular long stem with cementless fixation and interlocking screw fixation in the supracondylar area was considered [11]. This type of custom-made implant was used in 19 cases, mostly supported by an antibiotic-loaded allograft (• Fig. 2).

The use of structural allografts (preoperatively soaked in triple-loaded antibiotic solution) can reduce the rate of infection and instability in severe humeral bone loss and lead to a better functional outcome [8] even without any tendon transfer by healing of the soft tissues (mainly delta muscle and pectoralis major) to the graft. The allograft was fixed to the remaining diaphysis using either a telescoping or step-cut technique. At first, the so-called composite allograft fixation was used, in which the revision stem is cemented first to the allograft and later fixed to the diaphysis of the humerus by press fit or cement. Even with careful preoperative planning, this technique includes a higher risk of instability due to limited options for the final adjustment of humeral lengthening necessary for proper soft tissue tensioning. Filling of cavitary defects with cement is, in the authors' opinion, only useful in proximal humeral defects less than $5 \mathrm{~cm}$; with use of morselized bone graft now preferred instead.

\section{Discussion}

Only a few reports are available in the literature which focus on revisions of RSA, mainly from tertiary centers in France [6] or the USA $[14,28]$. To the best of the authors' knowledge, described herein is the largest series of revisions of RSA reported in the literature, even though there are several limitations. However, the purpose of this study was not the as- 
sessment of clinical outcome measured with patient-reported outcome measures (PROMs) or the Constant score, because it included treatment of a very inhomogeneous cohort. Instead, it was our goal to evaluate the failure mode characteristics and outcome parameters over a period of almost 10 years. In the literature, the clinical results of revision of RSA have been reported to be significantly inferior to primary RSA $[5,6,26,28]$, with high individual variation of the outcome related to the indication and pre-existing damage to the bone stock and soft tissue envelope.

The indications and risk factors identified herein are in line with the recently published data of the Danish multicenter study [2]. The risk of revision was higher in men and $33 \%$ of revisions were to treat periprosthetic infection. The only significant variable which influenced clinical outcome measured with the Western Ontario Osteoarthritis of the Shoulder (WOOS) score was the number of previous surgeries, which was considerably high in the present series: $25 \%$ of patients had undergone more than three revisions outside of the authors' hospital preoperatively.

In contrast to data given in metaanalyses of complications [3, 32], in the current series of patients who had been primarily operated on with numerous different designs, chronic periprosthetic infections (25\%) were the most frequent indication, more often than instability $(17.8 \%)$. The frequencies of humeral complications such as periprosthetic fractures (15.4\%), aseptic loosening $(11 \%)$, and septic stem loosening (18.3\%) were considerably higher. Aseptic glenoid loosening occurred in only $17 \%$, mostly in elderly female patients with very poor primary bone stock due to osteoporosis and excessive medialization or superior wear which was not corrected in the primary procedure by structural autograft rather than with an allograft or hardware augmentation.

In the literature, loosening of the baseplate is reported to occur in two peaks after implantation. The first peak occurs within the first 3 months postoperatively and the second in the mid- to long-term follow-up, mostly caused by progressive notching and excessive PE wear, which is rarely provoked by the use of a constrained ("retentive") PE liner today.

Stephens et al. [26] described a series of 85 patients who required revisions of RSA, previously operated on with a lateralized glenosphere design. The main indications for the revision were baseplate failures, infections, humeral dissociation, and glenosphere dissociation. Periprosthetic fractures, dislocations, and aseptic humeral loosening were rare. However, 18\% (15 of 85 ) required a subsequent re-revision. The most common failure mode in these patients was humeral dissociation $(n=6)$, followed by glenohumeral dislocation $(n=3)$, and periprosthetic fracture $(n=3)$. Salvage procedures were needed in 5 patients (6\%): 3 patients rejected a staged reimplantation and 2 patients required resection arthroplasty and conversion to hemiarthroplasty.

In 2016, Boileau et al. [5] reported different numbers for RSA with Grammont design from their tertiary center. In 60 revision cases (54 patients), they observed a considerably high number of instability cases, which were, with $38 \%$, more common than periprosthetic infections (22\%). Patients suffering from humeral complications (21\%) were revised more frequently than patients with glenoid loosening (13\%), which emphasizes the difference of complications between reverse and anatomical shoulder arthroplasty in mid- to long-term follow-up. However, in $10 \%$ of their patients, a salvage procedure (conversion to hemiarthroplasty or resection arthroplasty) was required.

In the past, instability was the most common cause of revision of RSA, with a wide range between 2.4 and $31 \%$. However, Boshali et al. [2] already calculated a $5 \%$ incidence of instability in their metaanalysis of publications between 2006 and 2015. Recently, the rate of dislocations in national registry data has dropped to less than $1 \%$ [18], but "subtle" instability caused by malposition of the baseplate is receiving more attention [27]. In the authors' experience, the intraoperative finding of tilting and subluxation was often related to preoperative complaints of pain, stiffness, and limited gain of active mobility.

While early dislocations within the first 3 months may be treated conservatively, late dislocations and chronic subluxations mostly require revision surgery. Our data confirm that the success rate drops with multiple redislocations, a prolonged delay of revision, and the number of failed surgical interventions $[3,4,13,23]$.

\section{Treatment algorithm in chronic instability}

An increase in soft tissue tension and higher compression load was first achieved through humeral lengthening using modular metallic components or higher PE inserts up to a maximum of $25 \mathrm{~mm}$, especially when the preoperative $\mathrm{CT}$ evaluation of humeral length did not reveal the value of at least $20 \mathrm{~mm}$ humeral lengthening which was previously recommended by Lädermann et al. as a result of their evaluation of instability cases [16].

Once remnants of the subscapularis were present, all efforts were directed towards mobilizing and reattaching the remaining soft tissues.

If more than $25 \mathrm{~mm}$ of humeral length had to be restored, this was first compensated by the additional exchange of the glenosphere to a larger diameter or an eccentric or lateralized design, in order to increase the bulk on the glenoid side. Finally, the stem was revised or exchanged to a proud position of at least $30 \mathrm{~mm}$ arm lengthening with neutral version. In case of chronic neurological lesions, especially neuropathy of the brachial plexus or complex regional pain syndrome (CRPS), excessive lengthening was avoided, because in the authors' experience, this implies the risk of deterioration of symptoms.

Dislocations related to medialization of the glenoid component were first corrected by the use of a lateralized or eccentric glenosphere of larger diameter and sometimes by complete exchange of the baseplate with a lateralizing autograft or allograft augmentation, preferably in a two-stage procedure because of the risk of baseplate avulsion after tight reposition. 


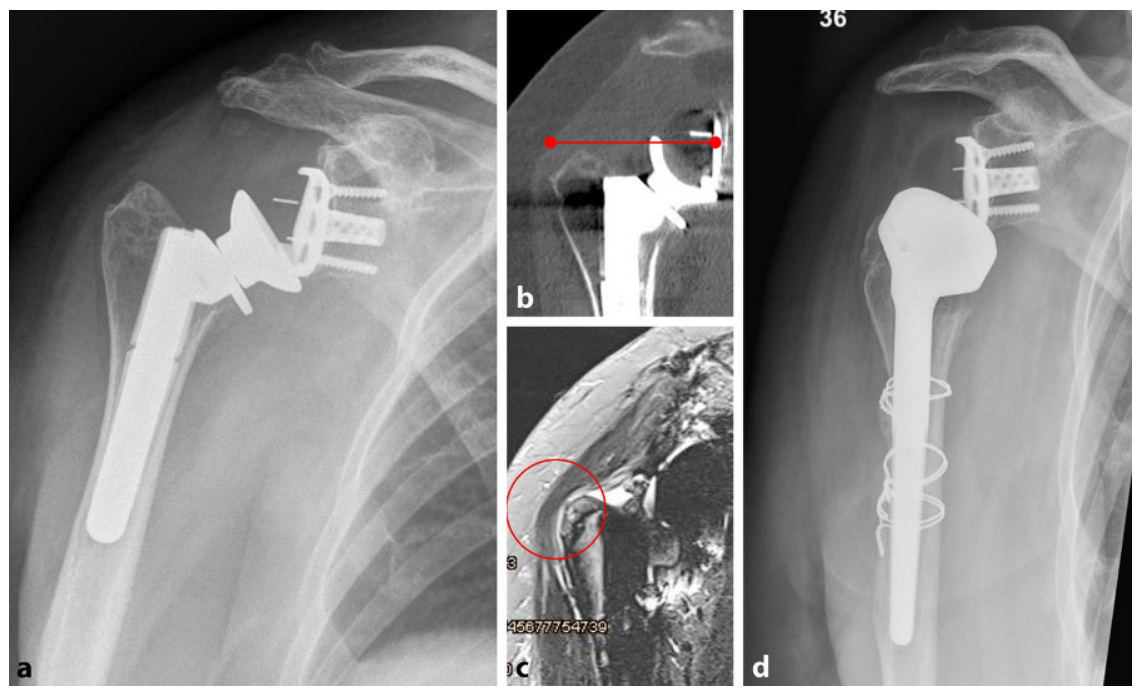

Fig. $4 \Delta$ Revision of a 68-year-old female patient suffering from very painful delta muscle wear, subtle instability, and pseudoparalysis caused by excessive humeral lateralization of an onlay platform design used in cuff tear arthropathy. a X-ray taken before revision. The company was asked for a custom-made tray in order to reduce the excessive lateralization of $44 \mathrm{~mm}$ but was unfortunately not able to deliver this component. A low-grade infection was excluded by arthroscopy which showed subluxation probably due to loss of compression load by the damage of the delta muscle. $b$ MRI shows thinning of the delta muscle by wear of the greater tuberosity; red line overall lateralization. C CT scan shows excessive lateralization caused by the design of the humeral component and bone ingrowth in the cementless fluted stem; red circle thinning of delta muscle. $\mathbf{d}$ Postoperative X-ray: conversion to RSA with medialized modular design of the stem and exchange to a larger glenosphere. The patient reported almost complete pain relief immediately after surgery. The active abduction improved gradually within the first 3 months to $80^{\circ}$

Wagner et al. [28] described the outcomes of 27 revisions of RSA with a mean follow-up of 4.4 years. The cause of failure of the primary RSA was mainly dislocation $(n=14,52 \%)$ and infection $(n=5,18.5 \%)$, whereas humeral-side failure, glenoid loosening, and periprosthetic humeral fractures were rare. Only 2 patients had combined glenoid loosening and dislocation. However, they observed complications in 11 patients $(41 \%)$, mainly recurrent instability $(n=5)$. Six cases $(22 \%)$ required further revision surgery and 5 patients suffering from dislocation $(n=3)$ and periprosthetic fractures $(n=2)$ were not revised. The high rate of glenoid lucency (23\%) was concerning.

Holcomb et al. analyzed the clinical outcome of 14 cases with RSA revised for baseplate loosening and found a rerevision rate of $14 \%$.

The rate of re-revision in cases with glenoid loosening in the current series was $20 \%$, higher than recently reported rates for revision of glenoid loosening in anatomical shoulder arthroplasty [12].
In the present RSA cases this was mainly caused by the delayed revision of the patients, who suffered from only little or moderate pain and therefore presented themselves too late, with advanced destruction of the bone stock and concomitant scapula spine or coracoid fatigue fracture (• Fig. 1).

Generally, the rate of complications and revisions of RSA used in revision shoulder arthroplasty is significantly higher compared to primary RSA, with a range from 5 to $45 \%[3,5,7,8$, $13,15,26,28,30,32]$. In the French multicenter study from 2016, Boileau et al. [6] described a re-revision rate in hemiarthroplasty converted to RSA of $20 \%$, from anatomical total shoulder arthroplasty to RSA of $10 \%$, and from RSA to RSA of $24 \%$.

Recently Knowles et al. [15] performed a systematic review of revisions of shoulder arthroplasty. The calculated complication rate for all revisions was $17 \%$. There was a total of 465 complications, of which $74 \%$ led to reoperations and re-revisions. Resection arthroplasty as a salvage procedure was only required in $2 \%$ of all complications. The most common indication for resection arthroplasty was instability (55\%).

The overall complication rate in the current study was $27 \%$ and the revision rate was $19.8 \%$, which was mainly influenced by the increase of humeral revisions and glenoid reconstructions with advanced bone loss. While the number of cases of glenosphere disassembly decreased, new hitherto unreported design failures related to onlay platform systems were observed (- Fig.4). Due to the shift to more demanding cases, which required new techniques and implants, a decrease in the number of complications was not observed.

\section{Conclusion}

The revision rate of $19.8 \%$ in the current series indicates that revision of RSA should be restricted to tertiary centers which have at least 5 years of experience with many different implant design types. Salvage procedures like resection arthroplasty or conversion to hemiarthroplasty were rarely needed (1.5\%), mostly due to severe comorbidity and excessive bone loss. A significant shift in indications and techniques according to new implant types of both the primary prosthesis and the revision implant was observed. Risk factors for further revision and unfavorable predictive outcomes are previous revision of chronic instability, severe glenoid and humeral bone loss exceeding $4^{\circ}$, recurrence of scapular spine fracture, and persisting chronic infection.

\section{Corresponding address}

Prof. Dr. Frank Gohlke
Klinik für Orthopädie,
Unfallchirurgie, Schulter-
chirurgie und Endoprothetik,
Rhön-Klinikum Campus Bad
Neustadt an der Saale
Von-Guttenberg-Str. 11,
97616 Bad Neustadt an der
Saale, Germany
frank.gohlke@campus-nes.de




\section{Compliance with ethical guidelines}

Conflict of interest. The senior author works as consultant for Wright/Tornier and is involved in the development of a modular revision implant without accepting royalties of any manufacturer or medical company.

All other authors have no conflict of interest.

All patients included in this retrospective evaluation have undersigned the written consent that all data including the clinical data and pre- and postoperative imaging can be used for medical education and scientific research including publication in medical literature.

Open Access This article is licensed under a Creative Commons Attribution 4.0 International License, which permits use, sharing, adaptation, distribution and reproduction in any medium or format, as long as you give appropriate credit to the original author(s) and the source, provide a link to the Creative Commons licence, and indicate if changes were made. The images or other third party material in this article are included in the article's Creative Commons licence, unless indicated otherwise in a credit line to the material. If material is not included in the article's Creative Commons licence and your intended use is not permitted by statutory regulation or exceeds the permitted use, you will need to obtain permission directly from the copyright holder. To view a copy of this licence, visit http://creativecommons.org/licenses/by/4.0/.

\section{References}

1. Ascione F, Kilian CM, Laughlin MS et al (2018) Increased scapular spine fractures after reverse shoulder arthroplasty with a humeral onlay short stem: an analysis of 485 consecutive cases. JShoulderElbow Surg 27:2183-2190

2. Baram A, Ammitzboell M, Brorson S, Olsen BS, Amundsen A, Rasmussen JV (2019) What factors are associated with revision or worse patientreported outcome after reverse shoulder arthroplasty for cuff-tear arthropathy? A study from the Danish shoulder arthroplasty registry. Clin Orthop Relat Res. https://doi.org/10.1097/CORR. 0000000000001114

3. Barco R, Savvidou OD, Sperling JW, SanchezSotelo J, Cofield RH (2016) Complications in reverse shoulder arthroplasty. EFORT Open Rev 1:72-80. https://doi.org/10.1302/2058-524.1.160003

4. Bohsali KI, Bois AJ, Wirth MA (2017) Complications of shoulder arthroplasty. J Bone Joint Surg Am 99:256-269

5. Boileau P (2016) Complications and revision of reverse total shoulder arthroplasty. Orthop Traumatol Surg Res 102:S33-S43

6. Boileau P, Chelli M, Favard L, Levigne C, Sirveaux $F_{\text {, }}$ Kempf JF, Clavert P, Collin P, Walch G (2016) The multicenter study: methodology \& presentation. In: Boileau P et al (ed) Reverse shoulder arthroplasty. Sauramps Medical, Montpellier

7. Casier S, Middernacht B, Van Tongel A, DeWilde L (2017) Revision of reversed shoulder arthroplasty. Is a reoperation possible? Obere Extremität 12:16-24. https://doi.org/10.1007/s11678-0170400-x
8. Chacon AVN, Shannon R, Levy J, Pupello D, Frankle M (2009) Revision arthroplasty with use of a reverse shoulder prosthesis-allograft composite. JBone Joint Surg Am 91:119-127

9. Favard L, Berhouet J, Walch G, Chaoui J, Lévigne C (2017) Superior glenoid inclination and glenoid bone loss: definition, assessment, biomechanica consequences, and surgical options. Orthopade 46(12):1015-1021. https://doi.org/10.1007/ s00132-017-3496-1

10. Gohlke F, Werner B (2009) Humeral bone defects in revision and primary shoulder arthroplasty. Proc. SECEC 2009, Madrid

11. Gohlke F, Werner B (2017) Humerale und glenoidale Knochendefekte in der Schulterendoprothetik: Klassifikation und Behandlungsprinzip ien [Humeral and glenoid bone loss in shoulder arthroplasty: classification and treatment principles]. Orthopade 46(12):1008-1014. https://doi org/10.1007/s00132-017-3484-5

12. Gohlke F, Werner B, Wiese I (2019) Glenoidrekonstruktion bei Wechseloperationen an der Schulter [Glenoid reconstruction in revision shoulder arthroplasty]. Oper Orthop Traumatol 31(2):98-114. https://doi.org/10.1007/s00064019-0594-8

13. Groh GI, Groh GM (2014) Complication rates, reoperation rates, and the learning curve in reverse shoulder arthroplasty. J Shoulder Elbow Surg 23(3):388-394. https://doi.org/10.1016/j.jse. 2013.06.002

14. Holcomb JO, Cuff D, Petersen SA, Pupello DR, Frankle MA (2009) Revision reverse shoulder arthroplasty for glenoid baseplate failure after primary reverse shoulder arthroplasty. J Shoulde Elbow Surg 18(5):717-723. https://doi.org/10. 1016/j.jse.2008.11.017

15. Knowles NK, Columbus MP, Wegmann K, Ferreira LM, Athwal GS (2020) Revision shoulder arthroplasty: a systematic review and comparison of North American vs. European outcomes and complications. J Shoulder Elbow Surg. https://doi. org/10.1016/j.jse.2019.12.015

16. Lädermann A, Williams MD, Melis B, Hoffmeyer $P$, Walch $G$ (2009) Objective evaluation of lengthening in reverse shoulder arthroplasty. J Shoulder Elbow Surg 18(4):588-595. https://doi.org/10. 1016/j.jse.2009.03.012

17. Lädermann A, Lübbeke A, Mélis B et al (2011) Prevalence of neurologic lesions after total shoulder arthroplasty. J Bone Joint Surg Am 93(14):1288-1293. https://doi.org/10.2106/JBJS. J.00369

18. Lehtimäki K, Rasmussen JV, Kukkonen J et al (2020) Low risk of revision after reverse shoulder arthroplasty for acute proximal humeral fractures. JSES Int 4(1):151-155. https://doi.org/10.1016/j. jses.2019.10.114

19. Levigne C, Garret J, Boileau P et al (2011) Scapular notching in reverse shoulder arthroplasty: is it important to avoid it and how? Clin Orthop Relat Res 469:2512-2520

20. Lowe JT, Lawler SM, Testa EJ, Jawa A (2018) Lateralization of the glenosphere in reverse shoulder arthroplasty decreases arm lengthening and demonstrates comparable risk of nerve injury compared with anatomic arthroplasty: a prospective cohort study. J Shoulder Elbow Surg 27(10):1845-1851. https://doi.org/10.1016/j.jse. 2018.06.018

21. Lucasti CJ, Namdari S (2019) Acromial stress fractures: a systematic review. Arch Bone Jt Surg 7(5):397-401
22. Melis B, Defranco M, Ladermann A et al (2011) An evaluation of the radiological changes around the Grammont reverse geometry shoulder arthroplasty after eight to 12 years. J Bone Joint Surg Br 93:1240-1246

23. Padegimas EM, Zmistowski B, Restrepo $C$, Abboud JA, Lazarus MD, Ramsey LM, Williams G, Namdari S (2016) Instability after reverse total shoulder arthroplasty: which patients dislocate? Am J Orthop 45(7):E444-E450

24. Ozgur SE, Sadeghpour R, Norris TR (2017) Revision shoulder arthroplasty with a reverse shoulder prosthesis. Orthopade 46(12):1055-1062. https:// doi.org/10.1007/s00132-017-3494-3

25. Scully WF, Wilson DJ, Parada SA, Arrington ED (2013) latrogenic nerve injuries in shoulder surgery. J Am Acad Orthop Surg 21(12):717-726. https://doi.org/10.5435/JAAOS-21-12-717

26. Stephens BC, Simon P, Clark RE, Christmas KN Stone GP, Lorenzetti AJ, Frankle MA (2016) Revision for a failed reverse: a 12-year review of a lateralized implant. J Shoulder Elbow Surg 25(5):e115-e124. https://doi.org/10.1016/j.jse.2015.09.027

27. Tashjian RZ, Martin BI, Ricketts CA, Henninger HB, GrangerEK, ChalmersPN (2018) Superiorbaseplate inclination is associated with instability after reverse total shoulder arthroplasty. Clin Orthop Relat Res 476(8):1622-1629. https://doi.org/10. 1097/CORR.0000000000000340Clin

28. Wagner ER, Hevesi M, Houdek MT, Cofield RH, Sperling JW, Sanchez-Sotelo J (2018) Can a reverse shoulder arthroplasty be used to revise a failed primary reverse shoulder arthroplasty?: Revision reverse shoulder arthroplasty for failed reverse prosthesis. bone Joint J 100-B(11):1493-1498. https://doi.org/10.1302/0301-620X.100B11.BJJ2018-0226.R2

29. Werner BS, Boehm D, Gohlke F (2013) Revision to reverse shoulder arthroplasty with retention of the humeral component. Acta Orthop 84(5):473-478 https://doi.org/10.3109/17453674.2013.842433

30. Werner BS, Boehm D, Hudek R, Plumhoff P, Burkhart KJ, Gohlke F (2016) Long-term analysis of revision reverse shoulder arthroplasty using cemented long stems. J Shoulder Elbow Surg 26(2):273-278. https://doi.org/10.1016/j.jse. 2016.05.015

31. Westermann RW, Pugely AJ, Martin CT, Gao $Y$, Wolf BR, Hettrich CM (2015) Reverse shoulder arthroplasty in the United States: a comparison of national volume, patient demographics, complications and surgical indications. lowa Orthop J J35:1-7

32. Zumstein MA, Pinedo M, Old J, Boileau P (2011) Problems, complications, reoperations, and revisions in reverse total shoulder arthroplasty: a systematic review. J Shoulder Elbow Surg 20:146-157 